\title{
A Modified Flanged Intrascleral Intraocular Lens Fixation Technique Using an Externalized Needle for Haptic Docking
}

\author{
Cristos Ifantides $\mathbb{D}^{1,2}$ \\ Steven M Naids ${ }^{3}$ \\ Danson V Muttuvelu (iD ${ }^{4}$ \\ Shahzad I Mian ${ }^{5}$ \\ Karen L Christopher (D) 1,6 \\ 'Department of Ophthalmology, Sue \\ Anschutz-Rodgers Eye Center, University \\ of Colorado School of Medicine, Aurora, \\ CO, USA; ${ }^{2}$ Department of Surgery, \\ Denver Health Medical Center, Denver, \\ CO, USA; ${ }^{3}$ Advanced Vision Care, Los \\ Angeles, CA, USA; ${ }^{4}$ Aarhus University \\ Hospital, Aarhus, Denmark; ${ }^{5}$ Department \\ of Ophthalmology and Visual Sciences, \\ W. K. Kellogg Eye Center, University of \\ Michigan, Ann Arbor, MI, USA; ${ }^{6}$ Rocky \\ Mountain Regional Veterans Affairs \\ Medical Center, Aurora, CO, USA
}

\begin{abstract}
The Yamane intrascleral flanged haptic fixation technique has obviated the need for resources such as suture or glue. However, intraocular maneuvers to properly dock haptics into the needles for externalization can be difficult for even adept eye surgeons and is especially difficult when visualization through the cornea is poor. Additionally, one traditional resource, intraocular forceps, has been critical in both the original technique and proposed modifications since its inception. We describe a modified flanged intrascleral intraocular lens fixation technique by docking the second haptic externally at the main corneal incision. This technique does not require the use of microforceps, which is advantageous to surgeons who lack access to specialized instrumentation. Additionally, this technique may provide added safety, visibility, and ease for surgeons by docking the haptic externally at the corneal incision rather than within the eye.

Keywords: secondary lens, secondary IOL, Yamane, haptic fixation, scleral fixation, cataract surgery, aphakia
\end{abstract}

\section{Introduction}

Since publication of the original Yamane technique using flanged intrascleral intraocular lens fixation via a double-needle technique, ${ }^{1}$ this sutureless procedure has gained popularity amongst both anterior segment and vitreoretinal surgeons. Fundamentally, his technique involves using two 30-gauge thin-wall needle to make angled sclerotomies $2 \mathrm{~mm}$ from the limbus 180 degrees apart from one another. Next, the leading haptic of a 3-piece intraocular lens is threaded into one needle lumen in the posterior segment using forceps, followed by trailing haptic into the other needle lumen. The haptics are then externalized and cautery is used to create a wider flange at the haptic tip. These are then settled back into the sclerotomies for stability by burying the flanged tips.

Advantages of this technique over other types of scleral fixation include lack of need for suture or glue, avoidance of conjunctival or scleral dissection, and small 30gauge sclerotomy size reducing risk of wound leak and hypotony. ${ }^{2}$ Several modifications on his original technique have been published, including a modification that we have adopted whereby the leading haptic is docked into a needle without the use of intraocular forceps. ${ }^{3-11}$ We describe a technique that is ideal for the anterior segment surgeon that may have less comfort docking haptics in the posterior segment. Additionally, this method avoids challenges associated with limited visualization of
Correspondence: Cristos Ifantides Department of Ophthalmology, Sue Anschutz-Rodgers Eye Center, University of Colorado, 1675 Aurora Court, F73I, Aurora, CO, 80045, USA

Email cristosMD@gmail.com 
the posterior segment and could potentially obviate the need for intraocular lens forceps.

\section{Methods}

In this technique, a conjunctival marking is made $2 \mathrm{~mm}$ away from the limbus and another $2 \mathrm{~mm}$ from the first parallel to the limbus. A second set of markings is created across the eye 180 degrees away from the original markings. A $1 / 2$ inch 30-gauge thin-walled needle (TSK ultra-thin wall needle; Tochigi Seiko, Tochigi, Japan) is bent approximately 70 degrees, $9 \mathrm{~mm}$ from the tip of the needle with the bevel facing towards the surgeon, similar to the "Injector-assisted haptic fixation for Yamane technique" originally described by Kim et al. ${ }^{11}$ A separate 1-inch 30-gauge thin-walled needle is bent approximately 70 degrees $20 \mathrm{~mm}$ from the tip of the needle with the bevel facing away from the surgeon. Adequate anterior vitrectomy should be performed via a limbal incision anterior vitrectomy, pars plana anterior vitrectomy, or complete pars plana vitrectomy.

A CT Lucia 3-piece lens (Carl Zeiss Meditec, Jena, Germany) is loaded into a 3-piece intraocular lens injector cartridge. A 2.75 bi- or tri-planar clear-corneal incision is made at the limbus, bisecting the two areas of markings. On the surgeon's left side, the $1 / 2$ inch needle is tunneled
$2 \mathrm{~mm}$ from the first marking towards the 180-degree marking (Figure 1.1) and then rotated and introduced into the eye (Figure 1.2). The lens injector cartridge is introduced into the eye through the $2.75 \mathrm{~mm}$ clear-corneal incision. The lens is advanced through the cartridge until the leading haptic has exited the cartridge. The injector is then rotated so the end of the leading haptic can be docked inside of the $1 / 2$ inch needle (Figure 1.3). Once the haptic is threaded into the needle, the intraocular lens is further advanced until it has exited the cartridge. The injector is then removed from the eye, leaving the trailing haptic to extend out of the main incision. Using the 1-inch needle, the surgeon tunnels through the sclera on the opposite side in a similar fashion to the first needle entrance (Figure 1.4). Once in the eye, the needle tip is advanced through the pupil and then externalized through the main incision, avoiding the creation of a false passage (Figure 1.5). Using forceps, the trailing haptic is placed inside the needle lumen (Figure 1.6). As the needle is drawn back into the eye, the haptic is further advanced into the needle lumen to ensure adequate docking. With both haptics docked in the needle lumens (Figure 1.7), the needles are slowly withdrawn out of the scleral tunnels (Figure 1.8). Using lowtemperature cautery, flanges are created at the haptic tips
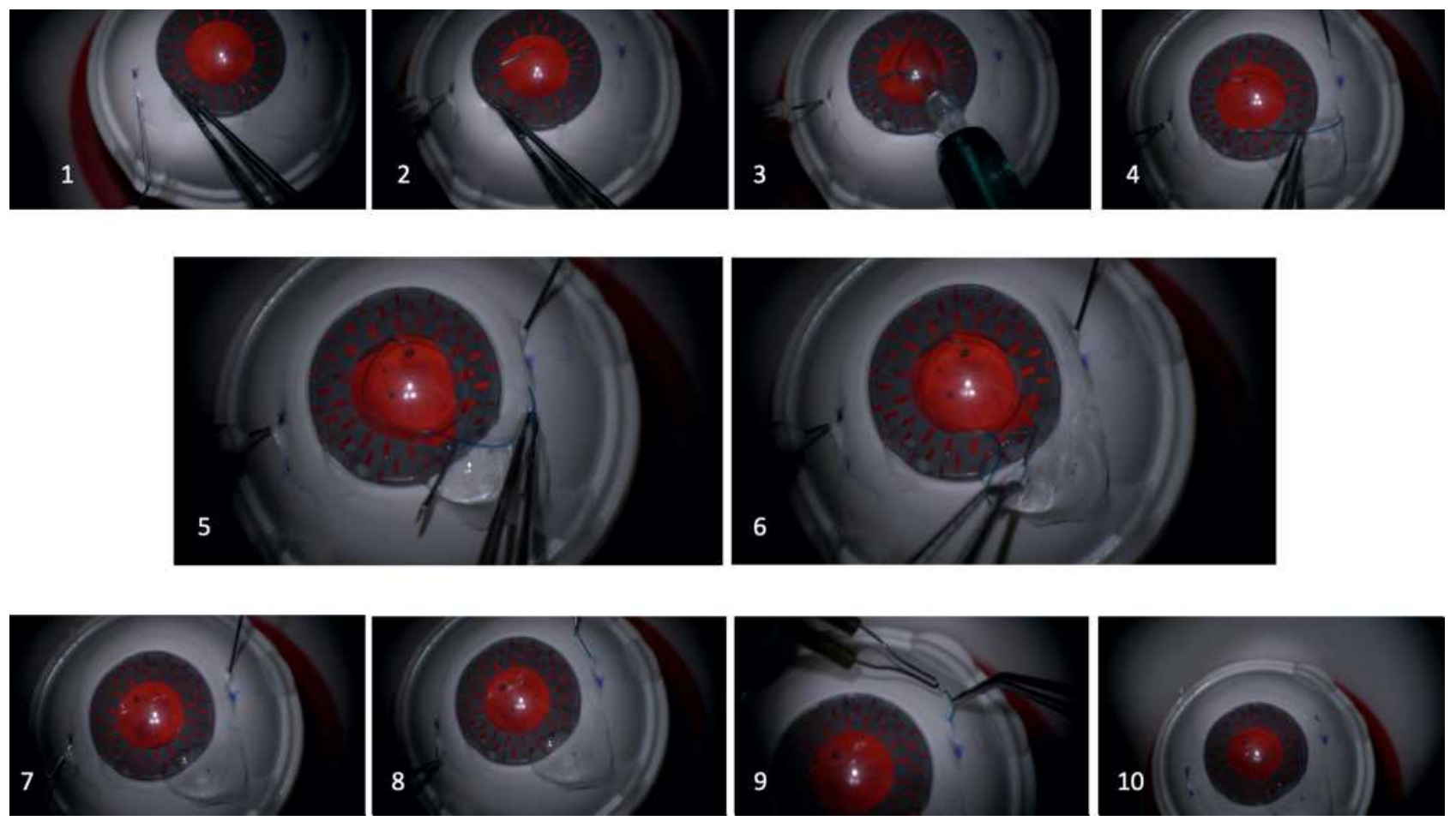

Figure I Step by step procedure in chronological order of externalized trailing haptic Yamane method on an artificial eye (Artificial eye is courtesy of Dr. Stuart Stoll and SimulEYE). 
(Figure 1.9) and the flanges are then securely seated back into the sclerotomies (Figure 1.10). A video guide on a model eye is provided, followed by intraoperative footage of the externalization technique of the distal haptic in a human eye (See Video). Video guide for step by step procedure of externalized trailing haptic Yamane method in an artificial eye, followed by use in a human eye. Some portions of the video are sped up $(2 \mathrm{x}-4 \mathrm{x})$ for viewer convenience (Artificial eye is courtesy of Dr. Stuart Stoll and SimulEYE). In this video, a 55-year-old patient with pseudoexfoliation syndrome was referred by a local ophthalmologist for hand motion vision and a severely luxated intraocular lens (IOL) in the left eye. Pertinent past ocular history included uncomplicated cataract surgery 6 years prior. Surgical repair was undertaken. Following vitrectomy, the IOL including the capsular bag was removed, and a 3-piece Zeiss Lucia $202 \mathrm{IOL}+22.0 \mathrm{D}$ was implanted in the anterior chamber, with the distal haptic docked externally using a wide-bore 30 gauge needle. The video demonstrates that the external technique for fixating the IOL is safe and no damage related to the main corneal wound or haptics was observed. Evaluating after one month the uncorrected VA was 6/7 and a well-centered IOL was observed.

\section{Conclusion}

Intrascleral intraocular lens fixation is an ideal technique for eyes which have absent or inadequate capsular support. Surgeons with poor visualization due to media opacity such as corneal scarring or edema, surgeons who have difficulty docking lens haptics into the needles in the posterior segment, and surgeons without access to intraocular microforceps may all look to this technique to provide a simple method for docking the intraocular lens haptics without the need of manipulation in the posterior segment.

The technique originally published by Yamane et al was an improvement on prior intrascleral lens fixation techniques. With this technique, there is no need for suture or glue to securely fixate the haptic to the sclera. Other pre-existing sutureless techniques use small tunnels from lamellar scleral dissections through which the distal haptic is threaded. ${ }^{12}$ While visual outcomes with techniques like this are similar to Yamane's flanged intrascleral fixation, surgeons have found the latter technique to be superior due to improved surgical time and lack of conjunctival cutdown. ${ }^{13}$ Furthermore, use of a smaller gauge needle to enter the sclera should reduce the risk of wound leak and post-operative hypotony, as well as provide a tight tunnel in which the haptic can dock for long-term stability. Additionally, haptic flanging confers more stability and a significantly higher required force to dislocate the haptic than non-flanged haptics. ${ }^{14}$

One of the difficult steps of Yamane's published technique is the docking of the second haptic into the needle. To avoid these risky intraocular movements, a trailinghaptic-first modification has been suggested to mitigate this risk. ${ }^{6}$ However, our technique of docking the second haptic into the needle externally from the corneal wound makes for a much simpler approach to overcome this obstacle. Additionally, because this step is performed outside of the eye, loss of corneal clarity or media opacity such as from hemorrhage in the eye no longer proves to be an impediment to accurately docking the haptics. The externalization technique of the distal haptic was performed in a total of 9 patients and no complication related to the IOL-implantation were observed. In 7 out of 9 patients the very flexible polyvinylidene difluoride (PVDF) haptic of the Zeiss CT Lucia IOLs was used, while in 2 patients, Johnson and Johnson (Jacksonville, Florida) 3-piece ZA9003 with PMMA haptics were utilized. While the PVDF haptics are very flexible, PMMA haptics must be handled more carefully. Particularly, no damage related to the bending of the haptics (PVDF or PMMA), or to the corneal wound was observed in any of these cases.

An intrascleral lens fixation technique that does not require the use of intraocular forceps is also appealing to surgical centers with limited resources, as well as surgeons who are less comfortable working with forceps in or near the posterior segment. Can et al also developed a technique that did not require forceps, and instead used a polytetrafluoroethylene polymer as a haptic guide. ${ }^{7}$ One centimeter of the guide is filled with a steel wire which allows it to be inserted through the sclera and externalized through the corneal incision, after which the haptic may be docked and the whole complex then externalized back through the sclera. While similar in concept of allowing the haptic docking to occur with ease external to the eye, our technique is advantageous over this technique in that it does not require this extra material of the polymer haptic guide.

We describe a modified flanged intrascleral intraocular lens fixation technique by docking the second haptic externally at the corneal incision. This technique allows surgeons to successfully dock the lens haptics even when 
there is loss of corneal clarity or other media opacity within the anterior segment. Additionally, many anterior segment surgeons may be more comfortable docking the haptic externally at the corneal incision rather than within the eye which may decrease damage to intraocular structures and improve surgical speed. Finally, this technique can obviate the need for expensive intraocular microforceps, which is advantageous for surgeons who may not have access to specialized instrumentation. Going forward, comparative studies on a larger group of patients and longer follow-up will be necessary to elucidate potential superiority of this presented surgical modification over the original Yamane fixation techniques or other known modifications.

\section{Disclosure}

The authors declare that they have no conflict of interest with the submitted manuscript.

\section{References}

1. Yamane S, Sato S, Maruyama-Inoue M, Kadonosono K. Flanged intrascleral intraocular lens fixation with double-needle technique. Ophthalmology. 2017;124(8):1136-1142. doi:10.1016/j. ophtha.2017.03.036

2. Jacob S, Kumar DA, Rao NK. Scleral fixation of intraocular lenses. Curr Opin Ophthalmol. 2020;31(1):50-60. doi:10.1097/ ICU.0000000000000632

3. Najafi M, Yamane S, Johnston RH. Modified flanged intrascleral fixation of intraocular lens for vitreoretinal surgeons. Ophthalmic Surg Lasers Imaging Retina. 2020;51(2):125-127. doi:10.3928/ 23258160-20200129-11

4. Diamint DV, Giambruni JM. 27-gauge trocar-assisted transconjunctival sutureless intraocular lens scleral fixation. Eur J Ophthalmol. 2020;1120672120919068. doi:10.1177/1120672120919068
5. Yamane S, Maruyama-Inoue M, Kadonosono K. Needle stabilizer for flanged intraocular lens fixation. Retina. 2019;39(4):801. doi:10.1097/IAE.0000000000002455

6. Kim DB. Trailing-haptic-first modification of double-needle intrascleral haptic fixation technique. J Cataract Refract Surg. 2018;44 (4):424-428. doi:10.1016/j.jcrs.2018.01.027

7. Can E. Flapless and sutureless intrascleral fixation of posterior chamber intraocular lens for correction of aphakia. $J$ Cataract Refract Surg. 2018;44(8):929-931. doi:10.1016/j.jcrs.2018.03.037

8. Ishikawa H, Fukuyama H, Komuku Y, Araki T, Gomi F. Flanged intraocular lens fixation via 27-gauge trocars using a double-needle technique decreases surgical wounds without losing its therapeutic effect. Acta Ophthalmol. 2020;98(4):e499-e503. doi:10.1111/ aos. 14313

9. Canabrava S, Canedo Domingos Lima AC, Ribeiro G. Fourflanged intrascleral intraocular lens fixation technique: no flaps, no knots, no glue. Cornea. 2020;39(4):527-528. doi:10.1097/ ICO.0000000000002185

10. Janse van Rensburg E, Ryu CL, Vila N, Chen JC. Sutureless intrascleral fixation of intraocular lens through self-sealing sclerotomy wounds using haptic externalization and reinternalization technique. $J$ Cataract Refract Surg. 2019;45(10):1367-1371. doi:10.1016/j. jcrs.2019.06.019

11. Kim DB. Yamane technique Kim method 1: injector-assisted haptic fixation, MA60AC, 27G 1/2 needle, SimulEye case. YouTube. 2020.

12. Yamane S, Inoue $M$, Arakawa A, Kadonosono K. Sutureless 27-gauge needle-guided intrascleral intraocular lens implantation with lamellar scleral dissection. Ophthalmology. 2014;121(1):61-66. doi:10.1016/j.ophtha.2013.08.043

13. Kelkar A, Kelkar J, Kothari A, et al. Comparison of two modified sutureless techniques of scleral fixation of intraocular lens. Ophthalmic Surg Lasers Imaging Retina. 2018;49(10):e129-e134. doi:10.3928/23258160-20181002-15

14. Stem MS, Wa CA, Todorich B, Woodward MA, Walsh MK, Wolfe JD. 27-gauge sutureless intrascleral fixation of intraocular lenses with haptic flanging: short-term clinical outcomes and a disinsertion force study. Retina. 2019;39(11):2149-2154. doi:10.1097/IAE.0000000000002268
Clinical Ophthalmology

\section{Publish your work in this journal}

Clinical Ophthalmology is an international, peer-reviewed journal covering all subspecialties within ophthalmology. Key topics include: Optometry; Visual science; Pharmacology and drug therapy in eye diseases; Basic Sciences; Primary and Secondary eye care; Patient Safety and Quality of Care Improvements. This journal is indexed on PubMed

\section{Dovepress}

Central and CAS, and is the official journal of The Society of Clinical Ophthalmology (SCO). The manuscript management system is completely online and includes a very quick and fair peer-review system, which is all easy to use. Visit http://www.dovepress.com/ testimonials.php to read real quotes from published authors. 